\title{
Factor XIII Deficiency, Review of the Literature and Report of a Case
}

\author{
Mojtaba Vahid Golpayegani, ${ }^{1}$ Hossein Behnia, ${ }^{2}$ Mahvareh Akhgar Araghi, ${ }^{3}$ and Ghassem Ansari ${ }^{1,}$ \\ ${ }^{1}$ Department of Pediatric Dentistry, School of Dentistry, Shahid Beheshti University of Medical Sciences, Tehran, IR Iran \\ ${ }^{2}$ Department of Oral \& Maxillofacial Surgery, School of Dentistry, Shahid Beheshti University of Medical Sciences, Tehran, IR Iran \\ ${ }^{3}$ Pedodontist, Tehran, IR Iran \\ "Corresponding author: Ghassem Ansari, Department of Pediatric Dentistry, School of Dentistry, Shahid Beheshti University of Medical Sciences, Tehran, IR Iran. Tel: +98-21 \\ 22255537, E-mail: profgansari@gmail.com
}

Received 2015 June 14; Revised 2016 July 24; Accepted 2016 August 12.

\begin{abstract}
Introduction: Factor XIII deficiency is a relatively rare hereditary bleeding disorder, which is usually found during infancy or early childhood. This condition may involve both genders within different races in an equal manner. Its incidence is estimated at approximately 1 in $10^{6}$ live births. Patients with factor XIII deficiency are presented with a pattern of neonatal hemorrhage and lifelong bleeding diathesis.

Case Presentation: A five-year-old female is presented here, who was diagnosed as a definite case of factor XIII deficiency, based on the clinical and laboratory findings at a medical specialist clinic. Typical clinical features of the disorder were the chief complaint of the patient, which was severe bleeding when she was brought into a specialized dental office following an earlier dental procedure. Conclusions: The case was fully investigated and diagnosed as a case with factor XIII deficiency causing such uncontrolled bleeding after extraction of a tooth.
\end{abstract}

Keywords: Factor XIII Deficiency, Coagulation Disorders, Bleeding Disorders, Clotting Problem

\section{Introduction}

Factor XIII deficiency (F XIII), the last enzyme in the coagulation cascade, is essential for normal homeostasis. It inhibits fibrinolysis and protects the clot from early breakdown (1). This factor, which is found in plasma, platelets and monocytes (2), is composed of two "A" subunits and two "B" subunits. Sakata et al. (3) first described FXIII deficiency in 1960 . Since this first description, up to 200 cases have been reported in the literature (4). Congenital FXIII deficiency is a rare disease but it is considered as one of the most severe hereditary bleeding disorders, with an estimated incidence of about 1 in 106. This disorder affects all races and both genders equally. It is inherited as an autosomal recessive trait, and usually manifests in life as early as infancy or early childhood (5). Most patients with inherited F XIII deficiency, experience severe and lifelong bleeding tendency with a high risk of death in early life because of intracranial hemorrhage.

Bleeding diathesis is severe in cases with inherited $\mathrm{F}$ XIII deficiency. The neonate with this disorder is at risk of bleeding immediately after birth (6). Umbilical bleeding in a newborn, which is considered as an important diagnostic clue, occurs in about $80 \%$ of the cases and is almost pathognomonic of F XIII deficiency (7). The Iranian tradition of circumcision within 24 hours of birth can lead to severe bleeding.

The incidence of intracranial hemorrhage is higher in
F XIII deficiency than in other inherited bleeding disorders (about 30\% of all cases). This represents an early lifethreatening factor and remains the main cause for the majority of deaths (8). These patients have a life-lasting tendency to bleed into the skin and subcutaneous tissues, which can cause extensive bruises. Bleeding into muscles and around joints are other common characteristics of this disorder, which could sometimes occur even after a minor trauma (6). Strenuous exercise can lead to bleeding in muscles, even in the absence of obvious local trauma. Bleeding from the gums during teething can become a serious problem. However, luckily bleeding from mucous membranes (i.e. epistaxis, gastrointestinal and renal hemorrhage) is a rare feature of this disorder (6).

A few patients have shown a tendency of mild bleeding, which can only be detected when a hemorrhagic procedure is attended, such as a surgical operation (9). Female patients with F XIII deficiency are unable to carry a pregnancy to term and present recurrent miscarriages (10). Wound healing problems have been reported in a minority of these patients (about 14\%) (9). The occurrence of keloid in these patients may be due to poor quality of clotting, which leads to abnormally large scar formation (11). This article aimed to elaborate clinical features of a young girl with such severe bleeding after a dental procedure. 


\section{Case Presentation}

A five-year-old female of middle-eastern origin was referred by a general dentist to a pediatric dental clinic for management of excessive bleeding. The problem started when her dentist conducted an incision at buccal vestibule of carious mandibular left primary second molar, to drain the abscess and reduce the swelling. The patient was pale on arrival to the dental clinic, with signs of malaise, agitation and fear. There was prominent swelling along with a large area of hematoma spanning anteriorly from the buccal vestibule of the first permanent molar, involving the mucus membrane of the lower lip and lingual of the lower left dentition. Crepitus feeling during palpation of the swelling together with oozing of blood from the clot was one of the main clinical findings (Figures 1 and 2).

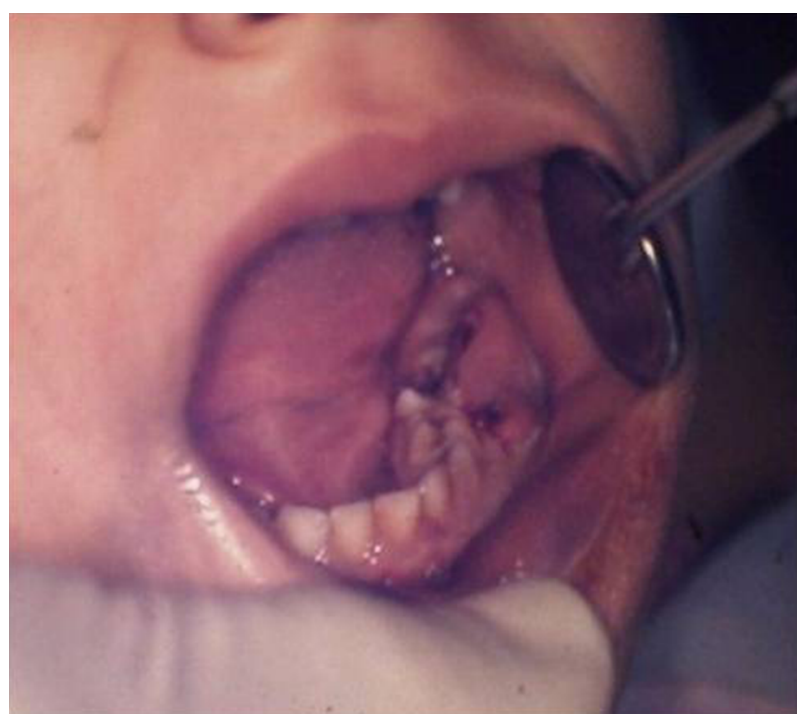

Figure 1. Swelling at the Buccal

Panoramic radiograph revealed deep carious lesion involving the pulp of the lower left second primary molar with no indication of forcation or apical pathology. Carious lesions involved other primary molars as well with some being treated before with amalgam. The panoramic radiograph also revealed the presence of a supernumerary tooth in the upper left central incisor region (Figure 3).

The patient was reportedly the only child of a nonconsanguine marriage. There was no trace of any family history of bleeding disorders. Postnatal history report revealed that umbilical bleeding had taken place, which had lasted up to two weeks. Other historical findings were negative except for occasional but minor ecchymosis due to trauma to her feet or hands.

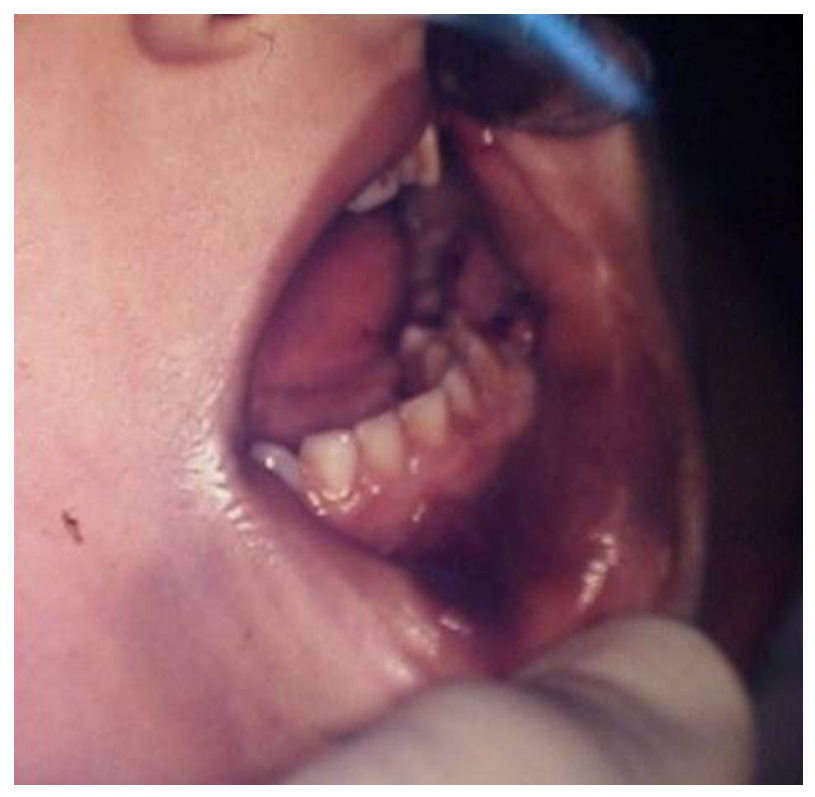

Figure 2. Extension Vestibule of the Left Second Primary Molar Hematoma in the Lower Lip

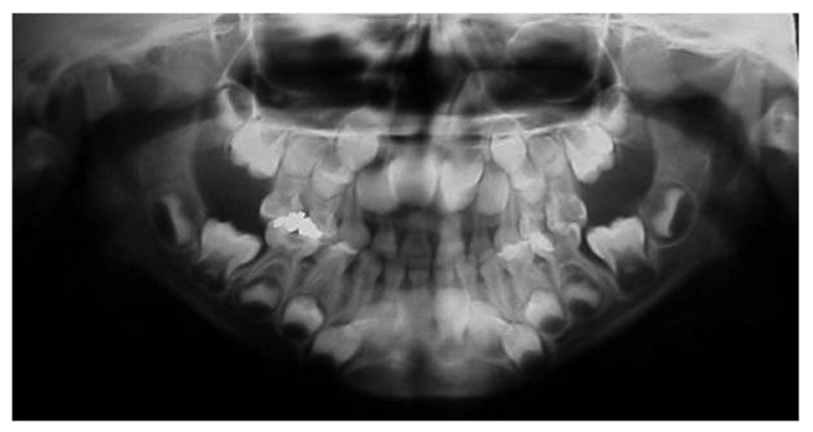

Figure 3. Panoramic Radiograph Showing Deep Carious Lesion in the Lower Left Second Primary Molar

\subsection{Management}

The patient was referred to a local children's hospital in order to control the bleeding. The following steps were carried out in line with bleeding control. An initial application of a sterile gauze pad over the extracted site after removing all the clots followed by an extra oral bandage of the face in order to apply pressure and control the bleeding (Figure 4).

Crystalloid solution was infused to substitute the amount of blood loss and to control the tachycardia. In the next step, a laboratory blood test was conducted. Hematologic examinations included the bleeding time (BT), prothrombin time (pt), partial thromboplastin time (PTT), and platelet count. All of which were found to be in the nor- 


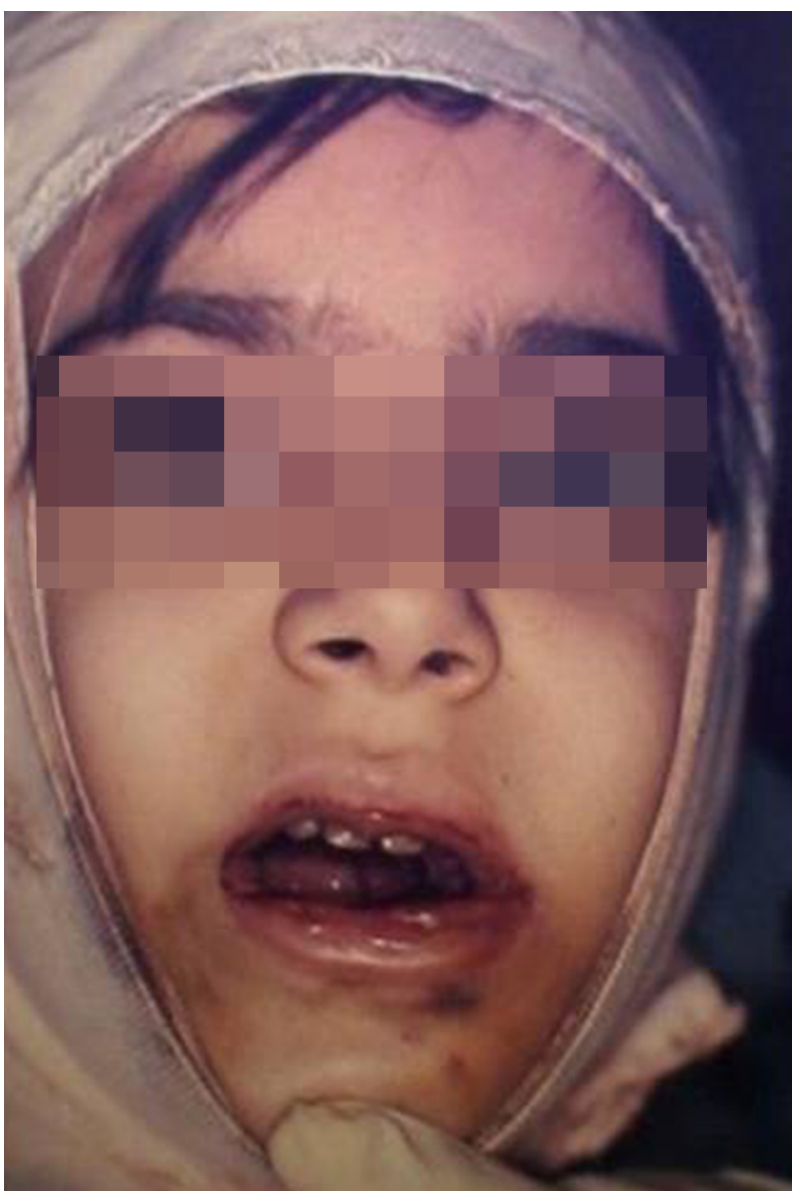

Figure 4. Patient With Extra-Oral Bandage

mal range. There was slight reduction in number of Red Blood Cells $\left(\mathrm{RBC}=3.8 \times 10^{3}\right)$, Hemoglobin $(\mathrm{HGB}=10.6 \mathrm{~g} / \mathrm{dL})$ and Hematocrit $(\mathrm{HCT}=31.0 \%)$, which could be explained by the excessive Blood loss. As the lab test results were not indicative of any clear bleeding disorder, the patient was hospitalized for further consultations and specific blood tests. Clot solubility test was then recommended at this stage for detection of a possible factor XIII problem. The result of the test revealed significant decrease in the level of Factor XIII (sub-unit A was less than $5 \%$ ).

On the basis of the clinical and laboratory findings, a diagnosis of factor XIII deficiency was eminent and confirmed by the hematologist. The patient subsequently received six bags of cryoprecipitate (containing F XIII) in order to obtain accurate homeostasis acceptable for such individual with severe bleeding from the oral cavity. If not controlled, it may have led to blood clot aspiration. Such preparation will allow further treatment on the subsequent days. The patient was discharged two days later with good general condition. Cryoprecipitate was prescribed for the patient, as a prophylactic measure, with an order of being administered prior to every medical or dental procedure with bleeding potentials. The patient was scheduled for a comprehensive dental treatment planning. Carious lesions were restored and Preventive Resin Restorations (PRR) were performed on several of the first permanent molars (Figures 5 and 6).

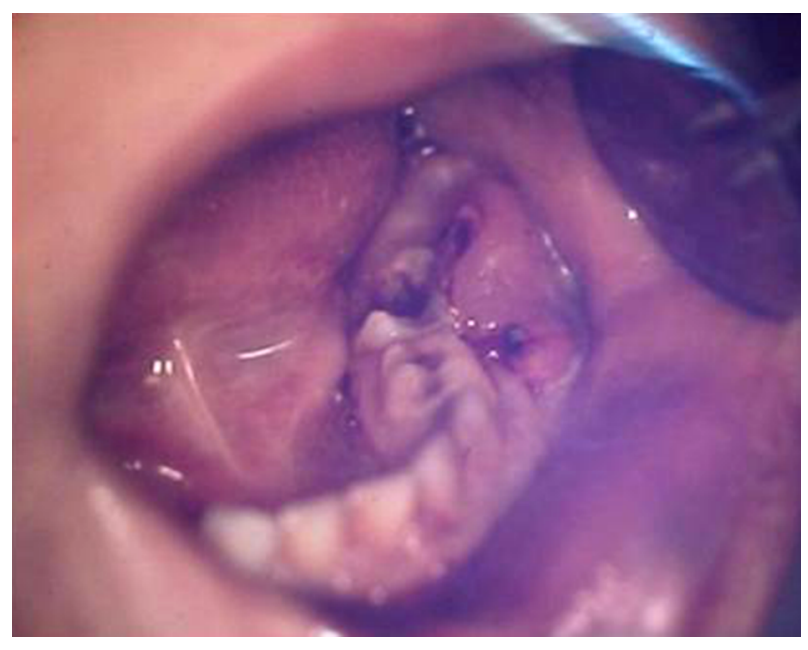

Figure 5. Hematoma and Inflammation

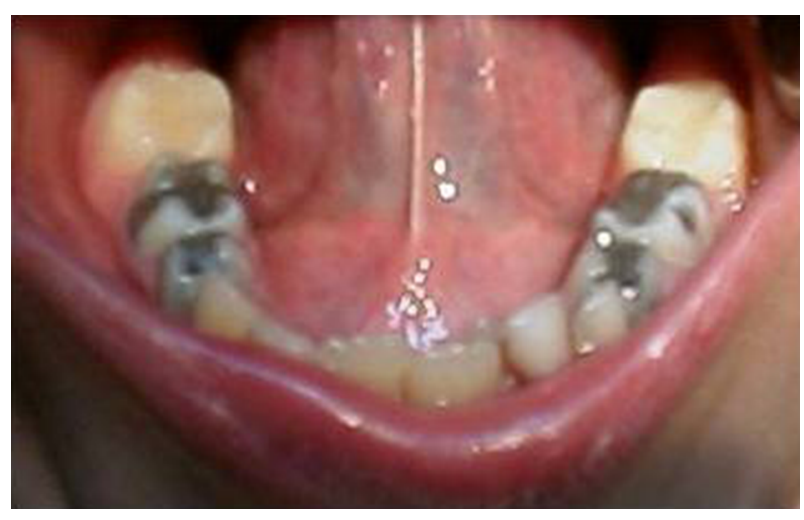

Figure 6. All Carious Primary Molars Disappeared. Emergency pulpotomy Were Restored and Preventive Resin was performed on the affected tooth Restorations Were done on 6's

A year and a half later, the mandibular primary central incisors were exfoliated with reportedly no bleeding complication. At seven years of age, the child returned with complaint of oozing gingival sulcus of loose maxillary primary centrals and mandibular primary laterals (Figure 7).

The involved teeth were scheduled for extraction and 


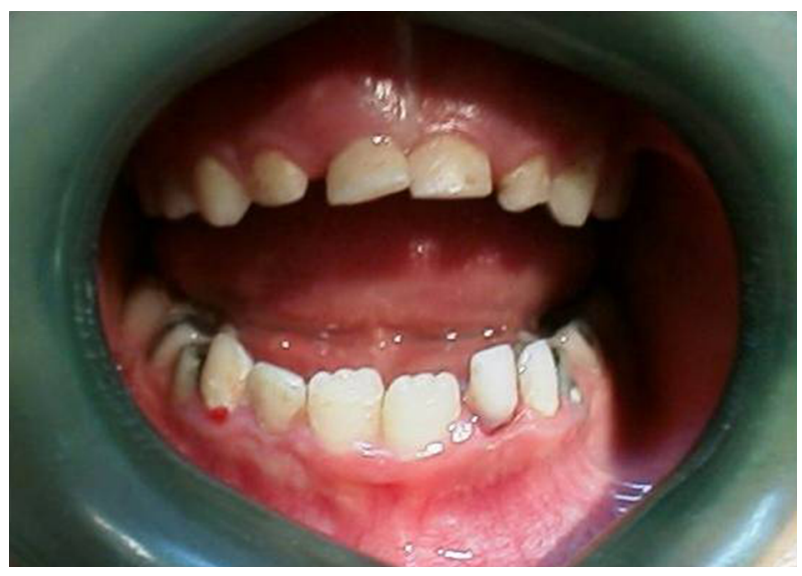

Figure 7. Presence of Blood Around Gingival Sulci of Lower Left Primary Lateral Incisor

the patient was referred to the hematologist for appropriate preparation. Teeth were then extracted without any Problem (Figures 8 and 9).

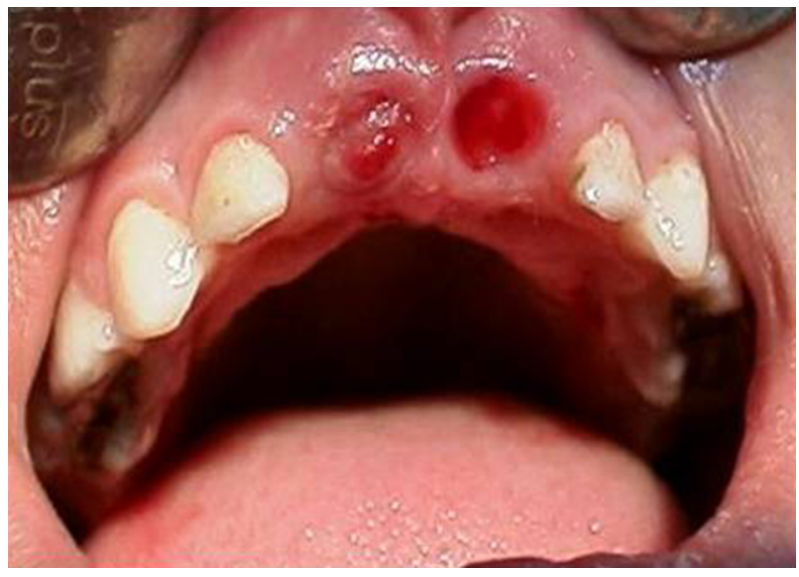

Figure 8. Extracted Sites of Maxillary

The supernumerary tooth was also scheduled at the same time for removal while it was still safe to do so. The patient was scheduled for a regular follow-up visit and proper dental and hematologic management, as this was believed to guarantee appropriate patient care.

\section{Discussion}

As standard clotting tests are normal in cases with $\mathrm{F}$ XIII deficiency, due to the clotting end point being unaffected by the absence of F XIII, their clinical diagnosis becomes highly critical. However, if noted from the qual-

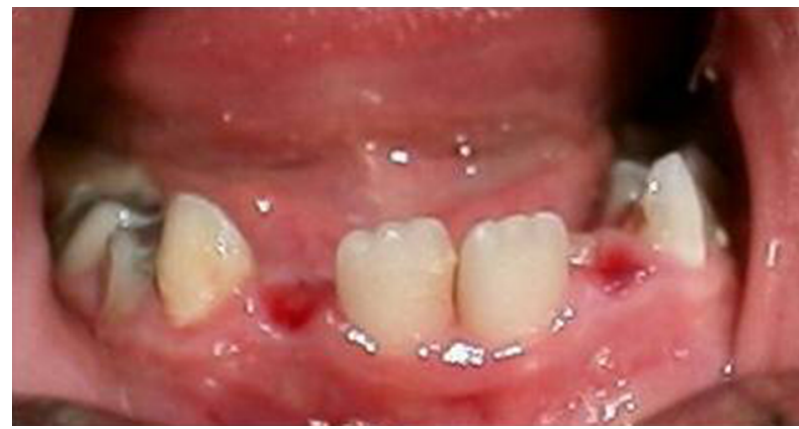

Figure 9. Extracted Sites of Mandibular Primary Central Incisors Primary Latera Incisors

ity of clot, which is abnormal, such entity will easily be identified and plenty of strategies can be arranged to prevent its complications. In these circumstances the diagnosis will not be missed when the quality of clotting is assessed (6). Standard clot solubility test is the screening test for F XIII deficiency in the urea and in some instances monochloroacetic acid measure (12). A definite diagnosis is then made on the basis of functional and immunological assays (7). For many years, whole blood, fresh frozen plasma or a placental F XIII concentrate have been used successfully in the treatment of F XIII deficiency. More recently, a plasma- derived pasteurized concentrate marketed under the name of Fibrogammin-P(Centeon Pharma $\mathrm{GmbH}$, Marburg, Germany) has also become available on the market. Since very low levels of F XIII in plasma are sufficient to control bleeding, and as the in vivo half-life of $\mathrm{F}$ XIII is long (11 - 14 days) after infusion, prophylaxis is considered as a simple and practical step (13). It is believed that any level of FXIII within 3 to $10 \%$ of the mean value in the normal population, i.e. $0.03-0.1 \mathrm{IU} / \mathrm{mL}$, is sufficient to prevent spontaneous hemorrhage (14-17). Although rare, $\mathrm{F}$ XIII deficiency is a remarkable disorder because of its serious bleeding manifestations. In particular, the majority of the cases have life-threatening symptoms early in life. However, there are reports of cases in the literature with problems that had not been detected until a later step and following severe bleeding as a sequel of a minor surgery (7).

The current patient was not diagnosed as an F XIII deficient case until the age of five years, despite prolonged umbilical bleeding during her neonatal period similar to the reported case by Anwar et al. (6). However, the key to early diagnosis is considered as the presence of serious bleeding manifestations, especially intracranial hemorrhage, which can easily be prevented if noted on time through prophylactic F XIII concentrate administration. 
Vigorous replacement therapy seems to be helpful in controlling the tendency of miscarriage in F XIII deficient pregnant females (6).

Decreased level of RBC*, $\mathrm{HGB}^{* *}$ and $\mathrm{HCT}^{* * *}$ may be due to excessive amount of blood loss after the first severe bleeding episode. A positive history of umbilical hemorrhage together with the clinical symptoms, led the hematologist to perform the F XIII assessment in order to confirm the diagnosis. As mentioned earlier, F XIII deficiency is an autosomal recessive disorder. This indicates that both parents must be carriers of the defective gene to pass it on to their children. In this patient, the negative familial and/or consanguinity history may attribute the disorder to mutations in F XIII type A gene. Recent studies have proved that such mutations are the most common causes of F XIII deficiency (15). Unlike this scenario, the case reported by Killick et al. (16) highlighted the importance of seeking familial history of bleeding disorders prior to surgery in the neonatal period, particularly if the parents are consanguineous.

Whole blood, fresh frozen plasma, stored plasma and cryoprecipitate are considered as the adequate sources of F XIII and have commonly been used successfully in the treatment of F XIII deficiency in the recent years (6). Fibrogammin-P is a recently available F XIII concentrate and is derived from the plasma of healthy donors. The methods of preparation of Fibrogammin-P have been described by Karges and Metzner (17). Patients are given 1000 units of concentrate every five to six weeks. A vial of concentrate contains 250 units, which is dissolved in $4 \mathrm{~mL}$ of water. Therefore, the patient receives $16 \mathrm{~mL}$ of the dissolved concentrate, intravenously for one to two minutes. This raises the plasma F XIII activity level to about 30-35\% of average normal levels while the process of coagulation remains normal for five to six weeks.

As young individuals are more active and therefore more prone to injury than adults, it has been recommended that they should receive the concentrate at fourweek intervals. However, Miloszewski and Losowsky (18) found six-week intervals as adequate in the prevention of hemorrhage in their patients. Although fibrogammin-P is the product of choice, due to its unavailability, cryoprecipitate (containing F I, F VIII, F XIII and Von Willebrand Factor) was infused to control bleeding. In order to control bleeding before the dental procedure Factor XIII 10 - 20 U/Kg concentrate was used as prophylactic therapy every four to six weeks in order to have adequate plasma level (19-22). As routine, it is recommended for all F XIII-deficient patients to receive prophylactic treatment immediately after confirmation of the diagnosis due to high risk of intracranial hemorrhage. From the dental management point, since hemorrhagic diseases are usually diagnosed at an early childhood stage, child dental clinics are possibly the first to see the manifestation of these disorders. In such cases, an accurate work up and correct laboratory tests and diagnosis could not only control and prevent the hemorrhage but also most of the dental procedures could be delivered to such individuals in a very safe and normal manner, similar to the case of this report.

\subsection{Conclusion:}

Patients with F XIII deficiency are uncommon, but could face serious life threatening issues following a simple dental procedure. It is important to note that umbilical hemorrhage does provide a diagnostic clue and should lead to a clot solubility test and F XIII assay early in life in order to make the parents and physicians including dentists, prepared for unexpected events.

\section{Acknowledgments}

The authors would like to express their sincere thanks and gratitude to Dr. M. Managhchi, Director of the hemophilia center at Imam hospital, Tehran University, for his valuable counseling and assistance during the course of controlling the hemorrhagic problem of the patient. Special thanks to Dr. Arash Vahid for his help in the preparation process of this manuscript.

\section{Footnote}

Authors' Contribution: Study concept and design: Mojtaba Vahid Golpayegani; acquisition of data: Mojtaba Vahid Golpayegani and Mahvareh Akhgar Araghi; analysis and interpretation of data: Mojtaba Vahid Golpayegani, Hossein Behnia and Ghassem Ansari; drafting of the manuscript: Mojtaba Vahid Golpayegani and Ghassem Ansari; critical revision of the manuscript for important intellectual content: Ghassem Ansari and Hossein Behnia; statistical analysis, administrative, technical and material support: Mojtaba Vahid Golpayegani and Mahvareh Akhgar Araghi ; study supervision: Mojtaba Vahid Golpayegani and Ghassem Ansari.

\section{References}

1. Aeschlimann D, Paulsson M. Transglutaminases: protein crosslinking enzymes in tissues and body fluids. Thromb Haemost. 1994;71(4):402-15. [PubMed: 7914385].

2. Muszbek L, Adany R, Szegedi G, Polgar J, Kavai M. Factor XIII of blood coagulation in human monocytes. Thromb Res. 1985;37(3):401-10. [PubMed: 2859670].

3. Sakata Y, Aoki N. Significance of cross-linking of alpha 2-plasmin inhibitor to fibrin in inhibition of fibrinolysis and in hemostasis. J Clin Invest. 1982;69(3):536-42. [PubMed: 7199538]. 
4. Board PG, Losowsky MS, Miloszewski KJ. Factor XIII: inherited and acquired deficiency. Blood Rev. 1993;7(4):229-42. [PubMed: 8130686].

5. Burrows RF, Ray JG, Burrows EA. Bleeding risk and reproductive capacity among patients with factor XIII deficiency: a case presentation and review of the literature. Obstet Gynecol Surv. 2000;55(2):1038. [PubMed: 10674253].

6. Anwar R, Miloszewski KJ. Factor XIII deficiency. Br J Haematol. 1999;107(3):468-84. [PubMed:10583246].

7. Anwar R, Minford A, Gallivan L, Trinh C, Markham AF. Delayed umbilical bleeding: A presenting feature for factor XIII deficiency: clinical features, genetics, and management. Pediatrics. 2002;109(2):32.

8. Miloszewski KJA, Losowski MS. In: Fibrin stabilization and factor XIII deficiency. Francis JL, editor. Ellis Horwood; 1988. pp. 175-202.

9. Lorand AL, Losowski MS, Miloszewski KJ. Human factor XIII: Fibrin stabilizing factor. ; 1980.

10. Kobayashi T, Terao T, Kojima T, Takamatsu J, Kamiya T, Saito H. Congenital factor XIII deficiency with treatment of factor XIII concentrate and normal vaginal delivery. Gynecol Obstet Invest. 1990;29(3):235-8. [PubMed: 2358199].

11. Al-Sharif FZ, Aljurf MD, Al-Momen AM, Ajlan AM, Musa MO, Al-Nounou $\mathrm{RM}$, et al. Clinical and laboratory features of congenital factor XIII deficiency. Saudi Med J. 2002;23(5):552-4. [PubMed:12070580].

12. Ragaz S, Kemp G, Furlan M, Beck EA. Bleeding disorder with abnormal wound healing, acid-soluble clots and normal factor XIII. Thromb Haemost. 1976;36(3):537-41. [PubMed: 1037151].

13. Cotter KL, Miloszewski KJ, Wall J, Losowsky MS. Effect of pasteurization on the placental factor XIII concentrate. BrJ Haematol. 1989;73(4):5745. [PubMed: 2611148].

14. Walls WD, Losowski MS. Congenital deficiency of fibrin-stabilizing factor. Coagulation. 1968;1:111-8.

15. Ichinose A, Souri M, Izumi T, Takahashi N. Molecular and genetic mechanisms of factor XIII A subunit deficiency. Semin Thromb Hemost. 2000;26(1):5-10. [PubMed: 10805274].

16. Killick CJ, Barton CJ, Aslam S, Standen G. Prenatal diagnosis in factor XIII-A deficiency. Arch Dis Child Fetal Neonatal Ed. 1999;80(3):238-9. [PubMed: 10212091].

17. Karges HE, Metzner HJ. Therapeutic factor XIII preparations and perspectives for recombinant factor XIII. Semin Thromb Hemost. 1996;22(5):427-36. doi: 10.1055/s-2007-999042. [PubMed: 8989827].

18. Miloszewski KJA, Losowsky MS, editors. Safety of long-term prophylaxis in inherited Factor XIII deficiency. Factor XIII. Second International Conference. Marburg, Stuttgart, Germany: FK Schattauer Verlagsgesellschaft mbH. 1993; pp. 155-60.

19. Dorgalaleh A, Naderi M, Hosseini MS, Alizadeh S, Hosseini S, Tabibian $S$, et al. Factor XIII deficiency in Iran: a comprehensive review of the literature. Semin Thromb Hemost. 2015;41(3):323-9. doi:10.1055/s-00341395350. [PubMed: 25615432].

20. Naderi M, Eshghi P, Cohan N, Miri-Moghaddam E, Yaghmaee M, Karimi M. Successful delivery in patients with FXIII deficiency receiving prophylaxis: report of 17 cases in Iran. Haemophilia. 2012;18(5):7736. doi: 10.1111/j.1365-2516.2012.02785.x. [PubMed: 22458944].

21. Inbal A, Oldenburg J, Carcao M, Rosholm A, Tehranchi R, Nugent D. Recombinant factor XIII: a safe and novel treatment for congenital factor XIII deficiency. Blood. 2012;119(22):5111-7. doi: 10.1182/blood-2011-10386045. [PubMed: 22451421].

22. Fadoo Z, Merchant Q, Rehman KA. New developments in the management of congenital Factor XIII deficiency. J Blood Med. 2013;4:65-73. doi: 10.2147/JBM.S32693. [PubMed: 23761984]. 